\title{
Note on Nordhaus-Gaddum problems for Colin de Verdière type parameters*
}

\author{
Wayne Barrett \\ Department of Mathematics \\ Brigham Young University \\ Provo, UT, USA \\ wayne@math. byu .edu \\ H. Tracy Hall \\ Department of Mathematics \\ Brigham Young University \\ Provo, UT, USA \\ H. Tracy@gmail.com
}

\author{
Shaun M. Fallat ${ }^{\dagger}$ \\ Department of Mathematics and Statistics \\ University of Regina, \\ Regina, SK, Canada \\ sfallat@math.uregina.ca \\ Leslie Hogben \\ Department of Mathematics \\ Iowa State University \\ Ames, IA, USA \\ American Institute of Mathematics \\ Palo Alto, CA, USA \\ LHogben@iastate.edu, hogben@aimath.org
}

Submitted: Jul 19, 2012; Accepted: Sep 24, 2013; Published: Oct 7, 2013

Mathematics Subject Classifications: 05C50, 05C40, 05C83, 15A03, $15 \mathrm{~B} 57$

\begin{abstract}
We establish the bounds $\frac{4}{3} \leqslant b_{\nu} \leqslant b_{\xi} \leqslant \sqrt{2}$, where $b_{\nu}$ and $b_{\xi}$ are the NordhausGaddum sum upper bound multipliers, i.e., $\nu(G)+\nu(\bar{G}) \leqslant b_{\nu}|G|$ and $\xi(G)+\xi(\bar{G}) \leqslant$ $b_{\xi}|G|$ for all graphs $G$, and $\nu$ and $\xi$ are Colin de Verdière type graph parameters. The Nordhaus-Gaddum sum lower bound for $\nu$ and $\xi$ is conjectured to be $|G|-2$, and if these parameters are replaced by the maximum nullity $\mathrm{M}(G)$, this bound is called the Graph Complement Conjecture in the study of minimum rank/maximum nullity problems.
\end{abstract}

Keywords: Nordhaus-Gaddum; Colin de Verdière type parameter; Graph Complement Conjecture; maximum nullity; minimum rank; graph complement

${ }^{*}$ This research began at University of Regina. The authors thank U. Regina and NSERC for their support.

${ }^{\dagger}$ Research supported in part by an NSERC research grant. 


\section{Introduction}

Nordhaus-Gaddum problems have been studied for many different graph parameters, including chromatic number, independence number, domination number, Hadwiger number, etc. (see, for example, [6] and the references therein). In this note we discuss the Nordhaus-Gaddum sum upper bounds for the Colin de Verdière type parameters $\nu$, $\xi$, and $\mu$. The Graph Complement Conjecture in the study of minimum rank/maximum nullity problems is a conjectured lower bound for related Nordhaus-Gaddum problems.

All graphs in this paper are simple, undirected, and finite. The complement of a graph $G=(V, E)$ is the graph $\bar{G}=(V, \bar{E})$, where $\bar{E}$ consists of all possible edges between vertices in $V$ that are not in $E$. Let $G$ be a graph with vertices $\{1, \ldots, n\}$ and let $S_{n}$ denote the set of symmetric $n \times n$ real matrices. For $A=\left[a_{i j}\right] \in S_{n}$, the graph of $A$, denoted by $\mathcal{G}(A)$, is the graph with vertices $\{1, \ldots, n\}$ and edges $\left\{i j: a_{i j} \neq 0\right.$ and $\left.i \neq j\right\}$. The set of symmetric matrices associated with $G$ is $\mathcal{S}(G)=\left\{A \in S_{n}: \mathcal{G}(A)=G\right\}$. The maximum nullity of $G$ is $\mathrm{M}(G)=\max \{$ null $A: A \in \mathcal{S}(G)\}$, and the maximum positive semidefinite nullity of $G$ is $\mathrm{M}_{+}(G)=\max \{$ null $A: A \in \mathcal{S}(G)$ and $A$ is positive semidefinite .

In [7] and [8], Colin de Verdière introduced the parameters $\mu(G)$ and $\nu(G)$, defined to be the maximum nullity among matrices $A \in \mathcal{S}(G)$ that satisfy the Strong Arnold Hypothesis and additional conditions. In [4] the parameter $\xi(G)$ was defined to be the maximum nullity among matrices $A \in \mathcal{S}(G)$ that satisfy the Strong Arnold Hypothesis. A real symmetric matrix $A$ satisfies the Strong Arnold Hypothesis provided there does not exist a nonzero real symmetric matrix $X$ satisfying $A X=0, A \circ X=0$, and $I \circ X=$ 0 , where $\circ$ denotes the Hadamard (entry-wise) product and $I$ is the identity matrix. The Strong Arnold Hypothesis is equivalent to the requirement that certain manifolds intersect transversally (see [13]). For $\nu$, the only additional condition (besides the Strong Arnold Hypothesis) is that the matrix must be positive semidefinite. For $\mu$, the additional conditions are that the matrix must be a generalized Laplacian (i.e., have nonpositive offdiagonal entries) and have exactly one negative eigenvalue. Clearly $\nu(G) \leqslant \mathrm{M}_{+}(G) \leqslant$ $\mathrm{M}(G), \nu(G) \leqslant \xi(G) \leqslant \mathrm{M}(G)$, and $\mu(G) \leqslant \xi(G)$, and each of these inequalities can be strict (see $[2])$.

An important property of Colin de Verdière type parameters is minor monotonicity. The contraction of edge $e=u v$ of $G$ is obtained by identifying the vertices $u$ and $v$, deleting any loops that arise in this process, and replacing any multiple edges by a single edge. A minor of $G$ arises by performing a sequence of deletions of edges, deletions of isolated vertices, and/or contractions of edges. The notation $H \preceq G$ means that $H$ is a minor of $G$. A graph parameter $\beta$ is minor monotone if for any minor $H$ of $G, \beta(H) \leqslant \beta(G)$. In [7], [8], and [4] (respectively) it is shown that $\mu, \nu$, and $\xi$ are minor monotone.

For any graph $G$, the Hadwiger number $\mathrm{h}(G)$ is the maximum order of a clique minor of $G$. It was shown in [7] and [8] that $\mu\left(K_{n}\right)=n-1$ and $\nu\left(K_{n}\right)=n-1$ (where $K_{n}$ denotes the complete graph on $n$ vertices), so by minor monotonicity $\mathrm{h}(G)-1 \leqslant \mu(G)$ and $\mathrm{h}(G)-1 \leqslant \nu(G)$.

Let $\kappa(G)$ denote the vertex connectivity of $G$, i.e., if $G$ is not complete, the smallest number $k$ such that there is a set of vertices $S$, with $|S|=k$, for which the graph obtained 
by deleting the vertices in $S$ and all edges incident with a vertex in $S$, denoted by $G-S$, is disconnected (by convention, $\kappa\left(K_{n}\right)=n-1$ ). It is proved in $[15,16]$ that $\kappa(G) \leqslant \mathrm{M}_{+}(G)$ for every graph $G$. It was noted in [12] that the proof in [15] establishes $\kappa(G) \leqslant \nu(G)$ for all $G$. As defined in [2], the minor monotone ceiling of $\kappa$ is $\lceil\kappa\rceil(G)=\max \{\kappa(H): H \preceq G\}$. It follows from the definition that $\mathrm{h}(G)-1 \leqslant\lceil\kappa\rceil(G)$, since the $K_{\mathrm{h}(G)}$ minor of $G$ implies $\kappa\left(K_{\mathrm{h}(G)}\right) \leqslant\lceil\kappa\rceil(G)$, and $\lceil\kappa\rceil(G) \leqslant \nu(G)$, since $\kappa(G) \leqslant \nu(G)$ and $\nu$ is minor monotone (see [2] for more detail).

A Nordhaus-Gaddum type result is a (sharp) lower or upper bound on the sum or product of a parameter of a graph and of its complement. The Graph Complement Conjecture for $\nu$ [3] is a Nordhaus-Gaddum sum lower bound.

Conjecture $1.1\left(\mathrm{GCC}_{\nu}\right)$. For any graph $G$,

$$
\nu(G)+\nu(\bar{G}) \geqslant|G|-2 .
$$

It is not possible to raise the lower bound $|G|-2$ since equality is attained for any tree that includes a $P_{4}$ : For such a tree, it is shown in [1] that $\mathrm{M}_{+}(\bar{T})=|T|-3$. Since $\mathrm{M}_{+}(T)=1, \mathrm{M}_{+}(T)+\mathrm{M}_{+}(\bar{T})=|T|-2$. It is shown in [17] that $\mathrm{GCC}_{\nu}$ is true for graphs with tree-width at most 3, and thus for trees. Thus $\mathrm{GCC}_{\nu}$ conjectures that $|G|-2$ is a tight Nordhaus-Gaddum sum lower bound for $\nu$. This conjecture is studied in [3], where it is established for certain graphs. Various other forms of this conjecture have appeared, including: $\mathrm{GCC}_{+}$, i.e., $\mathrm{M}_{+}(G)+\mathrm{M}_{+}(\bar{G}) \geqslant|G|-2$, [3]; GCC, i.e., $\mathrm{M}(G)+\mathrm{M}(\bar{G}) \geqslant|G|-2$, [5]; and $\mathrm{GCC}_{\xi}$, i.e., $\xi(G)+\xi(\bar{G}) \geqslant|G|-2$, [9]. Of course $\mathrm{GCC}_{\nu}$ implies $\mathrm{GCC}_{+}$implies GCC, and $\mathrm{GCC}_{\nu}$ implies $\mathrm{GCC}_{\xi}$ implies GCC. The graph complement conjecture for $\mu$, i.e., $\mu(G)+\mu(\bar{G}) \geqslant|G|-2$, appeared in [14].

Here we discuss values of the multiplier $b$ for a Nordhaus-Gaddum sum upper bound for the parameter $\beta$ where $\beta$ is one of $\mathrm{h},\lceil\kappa\rceil, \nu, \xi$, or $\mu$. We denote by $b_{\beta}$ the least value of $b$ making

$$
\beta(G)+\beta(\bar{G}) \leqslant b|G|
$$

true for every graph of order at least two, and call $b_{\beta}$ the $N G$ upper multiplier for $\beta$. Stiebitz [18] has shown that

$$
\mathrm{h}(G)+\mathrm{h}(\bar{G}) \leqslant \frac{6}{5}|G|
$$

and there exist graphs achieving $\mathrm{h}(G)+\mathrm{h}(\bar{G})=\frac{6}{5}(1-\varepsilon)|G|$ for arbitrarily small $\varepsilon$, so $b_{\mathrm{h}}=\frac{6}{5}$. We establish bounds for $b_{\lceil\kappa\rceil}, b_{\nu}$, and $b_{\xi}$. Clearly $b_{\mathrm{h}} \leqslant b_{\lceil\kappa\rceil} \leqslant b_{\nu} \leqslant b_{\xi}$, and $b_{\mathrm{h}} \leqslant b_{\mu} \leqslant b_{\xi}$. In Section 2 we construct a family of graphs to show that $b_{\lceil\kappa\rceil} \geqslant \frac{4}{3}$. In Section 3 we show that $b_{\xi} \leqslant \sqrt{2}$. In Section 4 we summarize our conclusions. Note that the Nordhaus-Gaddum sum upper bound for the parameters $\mathrm{M}$ and $\mathrm{M}_{+}$is not interesting because it is trivially $2|G|-1$ :

$$
\mathrm{M}\left(K_{n}\right)+\mathrm{M}\left(\overline{K_{n}}\right)=\mathrm{M}_{+}\left(K_{n}\right)+\mathrm{M}_{+}\left(\overline{K_{n}}\right)=(n-1)+n=2\left|K_{n}\right|-1 .
$$




\section{Lower bound for NG upper multiplier for $\lceil\kappa\rceil$}

In this section we construct a self-complementary graph $H(a)$ on $12 a-4$ vertices for $a \geqslant 2$, and show that $H(a)$ has a minor $\widehat{H(a)}$ with $\delta(\widehat{H(a)})=\kappa(\widehat{H(a)})=8 a-4$, where $\delta(G)$ denotes the minimum degree of a vertex of $G$. It is shown that this example implies that $b_{\lceil\kappa\rceil} \geqslant 4 / 3$.

Example 2.1. Construct the graph $H(a)=(V, E)$ as follows (see Figure 1): The $12 a-4$ vertices of $H(a)$ are partitioned into four sets $V_{i}, i=1,2,3,4$ of $r=3 a-1$ vertices each. The sets $V_{1}$ and $V_{2}$ are the "sparse part" of $H(a)$, with $H(a)\left[V_{i}\right]=\overline{K_{r}}, i=1,2$ (where $G[W]$ denotes the subgraph of $G$ induced by the subset $W$ of the vertices of $G$ ). The sets $V_{3}$ and $V_{4}$ are the "dense part" of $H(a)$, with $H(a)\left[V_{i}\right]=K_{r}, i=3,4$. Every edge between a vertex in $V_{1}$ and a vertex in $V_{3}$ is in the edge set $E$, and likewise for $V_{2}$ and $V_{4}$. There are no edges between $V_{1}$ and $V_{4}$, nor between $V_{2}$ and $V_{3}$. Regarding the edges between $V_{1}$ and $V_{2}$, number the vertices of $V_{1}$ as $u_{2 i-1}, i=1, \ldots, r$ and the vertices of $V_{2}$ as $u_{2 i}, i=1, \ldots, r$. Then vertex $u_{s} \in V_{1}$ is adjacent to the $a$ vertices $u_{s+j} \in V_{2}, j=1,3, \ldots, 2 a-3,2 a-1$ (where for $k=6 a-1, \ldots, 8 a-4, u_{k}$ is interpreted as $u_{\ell}$ with $\ell \equiv k \bmod (6 a-2)$ and $1 \leqslant \ell \leqslant 2 a-2$ ). If $u \in V_{1} \cup V_{2}$, then $\operatorname{deg}_{H(a)} u=r+a=4 a-1$ (where $\operatorname{deg}_{G} w$ denotes the degree of $w$ in $G$ ). Regarding the edges between $V_{3}$ and $V_{4}$, number the vertices of $V_{3}$ as $v_{2 i-1}, i=1, \ldots, r$ and the vertices of $V_{4}$ as $v_{2 i}, i=1, \ldots, r$. Then vertex $v_{s} \in V_{3}$ is adjacent to all vertices $v_{p} \in V_{4}$ except for $p=s+j, j=1,3, \ldots, 2 a-3,2 a-1$. If $v \in V_{3} \cup V_{4}$, then $\operatorname{deg}_{H(a)} v=(r-1)+r+(r-a)=8 a-4$. It is clear from the construction that $\overline{H(a)}=H(a)$.

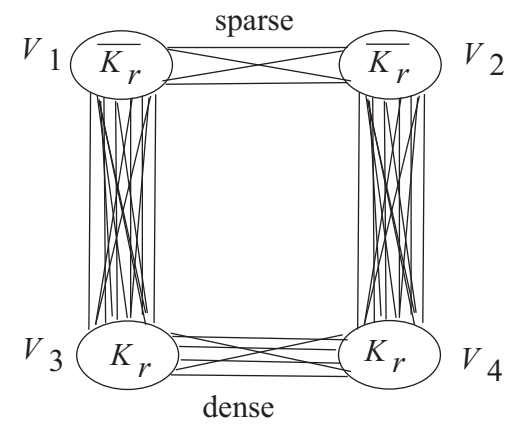

Figure 1: Schematic diagram for the construction of $H(a)$

Construct the minor $\widehat{H(a)}$ by contracting the edges $u_{2 i-1} u_{2 i}, i=1, \ldots, r$, and denote the set of these $r$ vertices by $V_{1,2}$. If $v \in V_{3} \cup V_{4}$, then $\operatorname{deg}_{\widehat{H(a)}} v=\operatorname{deg}_{H(a)} v=8 a-4$. Note that each of the new vertices in $V_{1,2}$ has degree equal to $2((4 a-1)-1)=8 a-4$, so $\widehat{H(a)}$ is $(8 a-4)$-regular. Furthermore, if $w \in V_{1,2}, w$ is adjacent to all $2 r=6 a-2$ vertices in $V_{3} \cup V_{4}$ so $\widehat{H(a)}\left[V_{1,2}\right]$ is $(2 a-2)$-regular. Since each vertex in $V_{3} \cup V_{4}$ is adjacent to $r=3 a-1$ vertices in $V_{1,2}, \widehat{H(a)}\left[V_{3} \cup V_{4}\right]$ is $(5 a-3)$-regular. 
To establish that $\kappa(\widehat{H(a)})=\delta(\widehat{H(a)})$, we use the property that for certain circulants $C$, $\kappa(C)=\delta(C)$, establish a method for computing $\kappa$, and examine parts of $\widehat{H(a)}$ separately. For $1 \leqslant t \leqslant\left\lfloor\frac{n-1}{2}\right\rfloor$, the consecutive circulant $\operatorname{Circ}_{n}\langle 1, \ldots, t\rangle$ is the graph on the vertices $\{0,1, \ldots, n-1\}$ with vertex $i$ adjacent to vertices $i+j$ and $i-j$ for $j=1, \ldots, t$ (with arithmetic $\bmod n$ ). We will use the fact that for a consecutive circulant the vertex connectivity is equal to the (common) degree; Harary [11] gave the consecutive circulant as an example of a graph having maximum vertex connectivity $\frac{2 m}{n}$ among graphs having $n$ vertices and $m$ edges (when $\frac{2 m}{n}$ is an integer), and this result is now well known.

Theorem 2.2. [19, Theorem 4.1.5 (Harary)] For $1 \leqslant t \leqslant\left\lfloor\frac{n-1}{2}\right\rfloor$,

$$
\kappa\left(\operatorname{Circ}_{n}\langle 1, \ldots, t\rangle\right)=\delta\left(\operatorname{Circ}_{n}\langle 1, \ldots, t\rangle\right)=2 t .
$$

Theorem 2.3. Let $G$ be a connected graph on $n$ vertices with $G \neq K_{n}$ and let $1 \leqslant t \leqslant$ $n-1-\delta(G)$. Define

$$
f(t)=\max \left\{s \in \mathbb{Z}^{+}: K_{t, s} \text { is a subgraph of } \bar{G}\right\}
$$

where $K_{s, t}$ denotes the complete bipartite graph on $s$ and $t$ vertices. Then

$$
\kappa(G)=\min \{n-(t+f(t)): 1 \leqslant t \leqslant n-1-\delta(G)\} .
$$

Proof. For every $t$ such that $1 \leqslant t \leqslant n-1-\delta(G), \bar{G}$ contains a $K_{t, 1}$ (by choosing a vertex $v$ of degree $\delta(G)$ as the partite set of 1 vertex, and $t$ of its non-neighbors as the other partite set), so $f(t)$ is defined.

Choose $t$ such that $1 \leqslant t \leqslant n-1-\delta(G)$. Let $U$ be a set of $t$ vertices and let $W$ be a set of $f(t)$ vertices such that $\bar{G}[U \cup W]$ contains a $K_{t, f(t)}$ subgraph. Then $G[U \cup W]$ is disconnected, so $\kappa(G) \leqslant|V \backslash(U \cup W)|=n-(t+f(t))$. Since this is true for every $t$ such that $1 \leqslant t \leqslant n-1-\delta(G)$,

$$
\kappa(G) \leqslant \min \{n-(t+f(t)): 1 \leqslant t \leqslant n-1-\delta(G)\} .
$$

Choose a set $S$ such that $|S|=\kappa(G)$ and $G-S$ is disconnected. Let $U$ be the set of vertices of one connected component, let $t_{0}=|U|$, and let $W=V \backslash(U \cup S)$; note $|W|=n-\left(t_{0}+\kappa(G)\right)$. Then $\bar{G}$ contains $K_{t_{0}, n-\left(t_{0}+\kappa(G)\right)}$ with bipartition $U, W$. Thus $f\left(t_{0}\right) \geqslant n-\left(t_{0}+\kappa(G)\right)$, so

$$
\kappa(G) \geqslant n-\left(t_{0}+f\left(t_{0}\right)\right) \geqslant \min \{n-(t+f(t)): 1 \leqslant t \leqslant n-1-\delta(G)\} .
$$

We now return to establishing the properties of one of the graphs constructed in Example 2.1.

Observation 2.4. Let $G$ be a graph whose vertex set $V$ can be partitioned as $V=X \dot{\cup} Y$ such that each vertex in $X$ is adjacent to each vertex in $Y$. Let $G_{X}=G[X]$ and $G_{Y}=$ $G[Y]$. Then 
- $\delta(G)=\min \left\{\delta\left(G_{X}\right)+|Y|, \delta\left(G_{Y}\right)+|X|\right\}$,

- $\kappa(G)=\min \left\{\kappa\left(G_{X}\right)+|Y|, \kappa\left(G_{Y}\right)+|X|\right\}$.

Theorem 2.5. For $\widehat{H(a)}$ as in Example 2.1,

$$
\kappa(\widehat{H(a)})=\delta(\widehat{H(a)})=8 a-4 .
$$

Proof. Let $X=V_{1,2}$ and $Y=V_{3} \cup V_{4}$. Then $V(\widehat{H(a)})=X \dot{\cup} Y$ and every vertex in $X$ is adjacent to every vertex in $Y$. By Observation 2.4, if we show that $\kappa\left(\widehat{H(a)}\left[V_{1,2}\right]\right)=$ $\delta\left(\widehat{H(a)}\left[V_{1,2}\right]\right)$ and $\kappa\left(\widehat{H(a)}\left[V_{3} \cup V_{4}\right]\right)=\delta\left(\widehat{H(a)}\left[V_{3} \cup V_{4}\right]\right)$, it follows that $\kappa(\widehat{H(a)})=\delta(\widehat{H(a)})$.

Since $\widehat{H(a)}\left[V_{1,2}\right]=\operatorname{Circ}_{r}\langle 1, \ldots, a-1\rangle$, we have $\kappa\left(\widehat{H(a)}\left[V_{1,2}\right]\right)=\delta\left(\widehat{H(a)}\left[V_{1,2}\right]\right)$ by Theorem 2.2 .

Recall that in $\widehat{H(a)}\left[V_{3} \cup V_{4}\right]=H(a)\left[V_{3} \cup V_{4}\right]$, the vertices of $V_{3}$ are numbered as $v_{2 i-1}, i=1, \ldots, r$ and the vertices of $V_{4}$ as $v_{2 i}, i=1, \ldots, r$, and vertex $v_{s} \in V_{3}$ is adjacent to all vertices $v_{p} \in V_{4}$ except for $p=s+j, j=1,3, \ldots, 2 a-3,2 a-1$ (where for $p>6 a-2, v_{p}$ means $\left.v_{p-(6 a-2)}\right)$. Thus the vertex $v_{s}$ in $\overline{H(a)\left[V_{3} \cup V_{4}\right]}$ is adjacent to precisely the vertices $v_{p}, p=s+j, j=1,3, \ldots, 2 a-3,2 a-1$. This is a bipartite regular graph with a great deal of symmetry, so in determining the shared neighborhood of two vertices, no generality is lost by considering the vertices 1 and $1+2 d$ (with $d \leqslant\left\lceil\frac{3 a-1}{2}\right\rceil$ ). The size of the shared neighborhood is $\max (a-d, 0)$. For $1 \leqslant t \leqslant a=2 r-1-(5 a-3)=\left|\widehat{H(a)}\left[V_{3} \cup V_{4}\right]\right|-1-$ $\delta\left(\widehat{H(a)}\left[V_{3} \cup V_{4}\right]\right)$, the maximum neighborhood intersection of a set of $t$ vertices happens when those vertices are consecutive in the same bipartition set, and it follows that in this case $f(t)=a+1-t$. So for all $t \in\{1, \ldots, a\},\left|\widehat{H(a)}\left[V_{3} \cup V_{4}\right]\right|-(t+f(t))=5 a-3$. Thus $\kappa\left(\widehat{H(a)}\left[V_{3} \cup V_{4}\right]\right)=5 a-3=\delta\left(\widehat{H(a)}\left[V_{3} \cup V_{4}\right]\right)$.

Corollary 2.6. For the graph $H(a)$ in Example 2.1,

$$
\lceil\kappa\rceil(H(a)) \geqslant 8 a-4
$$

and

$$
\lceil\kappa\rceil(H(a))+\lceil\kappa\rceil(\overline{H(a)}) \geqslant \frac{4}{3}\left(1-\frac{1}{6 a-2}\right)|H(a)| .
$$

Thus

$$
b_{\lceil\kappa\rceil} \geqslant \frac{4}{3} \text {. }
$$

Proof. By Theorem 2.5, \lceil\rceil$(H(a)) \geqslant \kappa(\widehat{H(a)})=8 a-4$. Since $H(a)$ is self-complementary, $\lceil\kappa\rceil(\overline{H(a)}) \geqslant 8 a-4$ also, and thus $\lceil\kappa\rceil(H(a))+\lceil\kappa\rceil(\overline{H(a)}) \geqslant 16 a-8$. The second statement can then be established by arithmetic. Since $b_{\lceil\kappa\rceil} \geqslant \frac{1}{|H(a)|}(\lceil\kappa\rceil(H(a))+\lceil\kappa\rceil(\overline{H(a)}))$, by taking the limit as $a \rightarrow \infty$ we see that $b_{\lceil\kappa\rceil} \geqslant \frac{4}{3}$. 


\section{Upper bound for NG upper multiplier for $\xi$}

In this section we show that the NG upper multiplier $b_{\xi}$ is at most $\sqrt{2}$.

Theorem 3.1. [10] Let $G=\left(V_{G}, E_{G}\right)$ be a connected graph. Then

$$
\left|E_{G}\right|+a \geqslant \frac{\xi(G)(\xi(G)+1)}{2}
$$

where $a=1$ if $G$ is bipartite and every optimal matrix for $\xi(G)$ has zero diagonal, and $a=0$ otherwise.

Since $\xi(G)$ is the maximum of $\xi\left(G_{i}\right)$ taken over the connected components $G_{i}$ of $G$, the hypothesis that $G$ is connected is unnecessary.

Corollary 3.2. Let $G=\left(V_{G}, E_{G}\right)$ be a graph. Then

$$
\left|E_{G}\right|+1 \geqslant \frac{\xi(G)(\xi(G)+1)}{2} .
$$

Corollary 3.3. Let $G=\left(V_{G}, E_{G}\right)$ be a graph with at least one edge. Then

$$
\xi(G) \leqslant \sqrt{2\left|E_{G}\right|}
$$

Proof. Algebraic manipulation of $(3)$ gives $\xi(G) \leqslant \frac{1}{2}\left(-1+\sqrt{8\left|E_{G}\right|+9}\right)$. Further manipulation shows that the inequality $\frac{1}{2}\left(-1+\sqrt{8\left|E_{G}\right|+9}\right) \leqslant \sqrt{2\left|E_{G}\right|}$ is equivalent to $2 \leqslant\left|E_{G}\right|$, so (4) is true if $G$ has at least two edges. If $G$ has exactly one edge then $G$ has components $K_{2}$ and possibly some $K_{1}$ 's, and thus $\xi(G)=1<\sqrt{2}$.

Nordhaus-Gaddum bounds usually take one of two forms: additive or multiplicative. The form of inequality (4) suggests a third category of Nordhaus-Gaddum bound: Pythagorean.

Corollary 3.4. Let $G=\left(V_{G}, E_{G}\right)$ be a graph of order at least two. Then

$$
\xi(G)^{2}+\xi(\bar{G})^{2} \leqslant|G|^{2}-|G| .
$$

Proof. Let $|G|=n \geqslant 2$. In the case where either $G$ has no edges or $\bar{G}$ has no edges, $\xi$ will take the value 1 for one of the two graphs and the value $n-1$ for the other, in which case the result holds. In any other case inequality (4) applies both to $G$ and to $\bar{G}$, giving us two inequalities the sum of whose squares is

$$
\xi(G)^{2}+\xi(\bar{G})^{2} \leqslant 2\left|E_{G}\right|+2\left|E_{\bar{G}}\right|=|G|^{2}-|G| .
$$

Corollary 3.5. Let $G=\left(V_{G}, E_{G}\right)$ be a graph of order at least two. Then

$$
\xi(G)+\xi(\bar{G}) \leqslant \sqrt{2}|G|
$$

and thus $b_{\xi} \leqslant \sqrt{2}$.

Proof. Let $|G|=n \geqslant 2$, and by Corollary 3.4 choose $x \geqslant \xi(G)$ and $y \geqslant \xi(\bar{G})$ such that $x$ and $y$ lie on the circle $x^{2}+y^{2}=n^{2}$. The maximum value of $x+y$ on this circle is $\sqrt{2} n$. 


\section{Conclusions}

In summary, we have established

$$
1.333<\frac{4}{3} \leqslant b_{\lceil\kappa\rceil} \leqslant b_{\nu} \leqslant b_{\xi} \leqslant \sqrt{2}<1.415 .
$$

We have no evidence that the construction in Section 2 is tight, even for $b_{\lceil\kappa\rceil}$. On the other hand, the inequality (2) with $a=0$ is known to be tight for some small examples and for complete graphs (it is tight with $a=1$ for $K_{3,3}$ ). For $\nu$, since a diagonal entry for a vertex of degree at least one cannot be zero, $a=0$ and the inequality (2) becomes $\left|E_{G}\right| \geqslant \frac{\nu(G)(\nu(G)+1)}{2}$ for graphs with at least one edge; again this is tight for some small graphs and complete graphs. This leaves open the possibility that Corollaries $3.3-3.5$ may be asymptotically tight.

Question 4.1. Given $x$ and $y$ positive with $x^{2}+y^{2}=1$, does there exist an increasing sequence of graphs $G_{i}$ on $n_{i}$ vertices such that $\nu\left(G_{i}\right) / n_{i}$ approaches $x$ and $\nu\left(\overline{G_{i}}\right) / n_{i}$ approaches $y$ ? Or such that $\xi\left(G_{i}\right) / n_{i}$ approaches $x$ and $\xi\left(\overline{G_{i}}\right) / n_{i}$ approaches $y$ ?

The particular case of $x=y=\frac{\sqrt{2}}{2}$ suggests the next question.

Question 4.2. Do $b_{\nu}$ and $b_{\xi}$ take the maximum possible value of $\sqrt{2}$ ?

On the other hand it seems more difficult to construct examples for $b_{\mu}$, and the only bounds we know are those from h (due to Stiebitz [18]) and $\xi$. i.e.,

$$
1.2=\frac{6}{5}=b_{\mathrm{h}} \leqslant b_{\mu} \leqslant b_{\xi} \leqslant \sqrt{2}<1.415 .
$$

\section{References}

[1] AIM Minimum Rank - Special Graphs Work Group (F. Barioli, W. Barrett, S. Butler, S. M. Cioabă, D. Cvetković, S. M. Fallat, C. Godsil, W. Haemers, L. Hogben, R. Mikkelson, S. Narayan, O. Pryporova, I. Sciriha, W. So, D. Stevanović, H. van der Holst, K. Vander Meulen, A. Wangsness). Zero forcing sets and the minimum rank of graphs. Linear Algebra Appl., 428: 1628-1648, 2008.

[2] F. Barioli, W. Barrett, S. Fallat, H. T. Hall, L. Hogben, B. Shader, P. van den Driessche, H. van der Holst. Parameters related to tree-width, zero forcing, and maximum nullity of a graph. J. Graph Theory, 72: 146-177, 2013.

[3] F. Barioli, W. Barrett, S. M. Fallat, H. T. Hall, L. Hogben, H. van der Holst. On the graph complement conjecture for minimum rank. Linear Algebra Appl., 436: 43734391, 2012.

[4] F. Barioli, S. M. Fallat, and L. Hogben. A variant on the graph parameters of Colin de Verdière: Implications to the minimum rank of graphs. Electron. J. of Linear Algebra, 13: 387-404, 2005. 
[5] R. Brualdi, L. Hogben, B. Shader. AIM Workshop on Spectra of Families of Matrices Described by Graphs, Digraphs and Sign Patterns, Final report: Mathematical Results, 2007. http://aimath.org/pastworkshops/matrixspectrumrep.pdf.

[6] G. Chartrand and J. Mitchem. Graphical theorems of the Nordhaus-Gaddum class. In Recent Trends in Graph Theory, Lecture Notes in Mathematics 186, Springer, Berlin, pp. 55-61. 1971.

[7] Y. Colin de Verdière. On a new graph invariant and a criterion for planarity. In Graph Structure Theory, Contemporary Mathematics 147, American Mathematical Society, Providence, pp. 137-147, 1993.

[8] Y. Colin de Verdière, Multiplicities of eigenvalues and tree-width of graphs, J. Combin. Theory Ser. B, 74: 121-146, 1998.

[9] S. Fallat and L. Hogben. Minimum Rank, Maximum Nullity, and Zero Forcing Number of Graphs. In Handbook of Linear Algebra, 2nd ed., CRC Press, Boca Raton, FL, 2013.

[10] H. T. Hall, L. Hogben, R. Martin, B. Shader. Expected values of parameters associated with the minimum rank of a graph. Linear Algebra Appl., 433: 101-117, 2010.

[11] F. Harary. The maximum connectivity of a graph. Proc. National Academy Sciences, 48: 1142-1146, 1962.

[12] H. van der Holst. Three-connected graphs whose maximum nullity is at most three. Linear Algebra Appl., 429: 625-632, 2008.

[13] H. van der Holst, L. Lovász, and A. Schrijver, The Colin de Verdière graph parameter. In Graph Theory and Computational Biology (Balatonlelle, 1996), Bolyai Soc. Math. Stud. 7, János Bolyai Math. Soc., Budapest, pp. 29-85, 1999.

[14] A. Kotlov, L. Lovász, S. Vempala. The Colin de Verdère number and sphere representations of a graph. Combinatorica, 17: 483-521, 1997.

[15] L. Lovász, M. Saks and A. Schrijver. Orthogonal representations and connectivity of graphs. Linear Algebra Appl., 114/115: 439-454, 1989.

[16] L. Lovász, M. Saks and A. Schrijver. A correction: "Orthogonal representations and connectivity of graphs." Linear Algebra Appl., 313: 101-105, 2000.

[17] J. Sinkovic and H. van der Holst. The minimum semidefinite rank of the complement of partial k-trees. Linear Algebra Appl., 434: 1468-1474, 2011.

[18] M. Stiebitz. On Hadwiger's number - a problem of the Nordhaus-Gaddum type. Discrete Math., 101: 307-317, 1992.

[19] Douglas B. West. Introduction to Graph Theory, 2nd ed., Prentice Hall, New Jersey, 2001. 